\title{
Aldo Nove, de Woobinda à Superwoobinda : l'éthique du trash et ses limites
}

Martine Bovo-Romoeuf

\section{OpenEdition}

Journals

Édition électronique

URL : http://journals.openedition.org/cei/107

DOI : $10.4000 /$ cei. 107

ISSN : 2260-779X

Éditeur

UGA Éditions/Université Grenoble Alpes

Édition imprimée

Date de publication : 15 juin 2010

Pagination : 73-89

ISBN : 978-2-84310-168-7

ISSN : 1770-9571

Référence électronique

Martine Bovo-Romoeuf, "Aldo Nove, de Woobinda à Superwoobinda : l'éthique du trash et ses limites ", Cahiers d'études italiennes [En ligne], 11 | 2010, mis en ligne le 15 décembre 2011, consulté le 27 mars 2021. URL : http://journals.openedition.org/cei/107 ; DOI : https://doi.org/10.4000/cei.107 


\title{
ALDO NOVE, DE WOOBINDA À SUPERWOOBINDA : L'ÉTHIQUE DU TRASH ET SES LIMITES
}

\author{
Martine Bovo \\ Université de Bordeaux
}

«Sapessi cosa ti sei persa!»

C'est en ces termes qu'Ugo Tognazzi, dans un célèbre épisode des Mostri de Dino Risi (1963), incarnant un mari pantouflard, drapé confortablement dans sa robe de chambre, le nez chaussé de lunettes aussi épaisses qu'un fond de bouteille, s'adressait à sa femme, la sémillante et coquette Michèle Mercier. La saynète, intitulée L'oppio dei popoli mettait en place dès 1963 cette image comique du teledipendente: Ugo Tognazzi passait toute la soirée assis les yeux rivés à l'écran du téléviseur pendant que sa charmante épouse, sachant que rien au monde ne l'en éloignerait, recevait en toute impunité son amant dans la pièce voisine. L'émission terminée, après avoir regardé avidement tout le programme de la soirée même les dernières images annonçant la fin des programmes, Tognazzi regagnait la chambre conjugale, dont venait de sortir en toute hâte l'amant, et prononçait avec une pointe de regret la phrase mémorable.

La télévision, apparue dès janvier 1954 dans les foyers italiens ${ }^{\mathrm{I}}$ fait partie des grands mythes ayant accompagné l'avènement du boom économique de la péninsule : à la fois symbole du nouveau bien-être social du pays et d'un confort privé et familial fraîchement acquis, elle fut rapidement brocardée par le cinéma italien et notamment par les grands noms de la commedia all'italiana (Giacovelli, 1995) qui contribuèrent non seulement

I. Les premières émissions télévisées sont apparues en janvier I954 et ce n’est qu’en I957 qu’elles seront diffusées sur le plan national. 
à diffuser son mythe mais aussi à le regarder avec la distance et le détachement caractéristiques des meilleures productions humoristiques. Dans son film, Dino Risi ne lésinait pas sur la puissance de la représentation : L'oppio dei popoli était suivi d'une autre saynète qui s'intéressait cette fois non plus à ce qui se passait du côté des spectateurs mais du côté des acteurs. Après le tele-schiavo, elle nous livrait le portrait du tele-divo dans Il testamento di San Francesco où le prêtre, interprété par Vittorio Gassmann, prêchait avec passion et véhémence au petit écran amour et renoncements franciscains alors que dans la réalité des coulisses, enfin montré sous son véritable jour, il apparaissait plus préoccupé de son maquillage et de son apparence que de la charité chrétienne qui avait tant nourri ses discours enflammés. Quelques années plus tard, en 1966, c'était le tour de Pasolini de dénoncer la fonction niveleuse de la télévision, "cette machine de vulgarité et de mesquinerie», son conformisme des plus dangereux qui trouvait alors un terrain propice chez les petits-bourgeois et les classes moyennes ${ }^{2}$.

Notre intention n'est pas ici de traiter du rapport entre le cinéma des années 1960 et la télévision mais de repérer les jalons d'un rapport à la fois critique et satirique à l'image du réel que l'univers cathodique a pu restituer et imposer à la population. Les deux saynètes de Dino Risi précédemment évoquées, ponctuées de musiques rythmées, dénonçaient déjà l'asservissement de l'individu à la télévision, fustigeaient cette fascination pouvant priver de la raison la plus élémentaire (dans le premier cas) pour voir la réalité des faits, ou pouvant conduire à la manipulation du réel et à la vanité (dans le second cas). Cette dénonciation se déclinait toutefois sur le registre du comique de situation et sur une capacité très italienne de se moquer de soi, de savoir avec élégance et humour tourner en dérision les travers de personnages représentatifs des valeurs auxquels les Italiens demeuraient attachés.

De nombreux points nous poussent à voir un prolongement de cette tradition dans la production narrative d'Aldo Nove, si ce n'est qu'il ne s'agit pas d'une continuité à proprement parler, mais plutôt d'un dévoiement de cette capacité à se moquer de soi, repérable à travers l'analyse du recueil Woobinda, (Nove, I996), suivi deux ans plus tard par Superwoobinda.

2. "Puisqu'il y a un mois que je suis malade assez gravement, il y a un mois que je suis à la maison et donc un mois que tous les soirs, ne pouvant pas lire, je regarde la télévision. C'est infiniment pire et plus dégradant que ce que la plus féroce imagination peut supposer. " (Pasolini, 2003, p. II.) 


\section{Woobinda}

L'objet de la satire de Nove est l'Italie des années 1980-1990 avec les égéries télévisées qui ont contribué à forger l'imaginaire collectif de toute une génération, Maurizio Costanzo, Mara Venier, Iva Zanicchi e Magalli... mais c'est surtout une humanité apocalyptique que nous présente l'auteur dans les micro-récits de Woobinda. Le choix formel de l'ouvrage est en soi intéressant puisque l'objet/livre devient délibérément un clone de l'objet/télé : même composition interne à partir de micro-séquences narratives organisées en lots (que l'auteur numérote soigneusement jusqu'au huitième), toujours brèves (comme des spots publicitaires), dont le cours est interrompu comme si la plume de l'écrivain opérait un zapping télévisé (certains récits sont interrompus brutalement comme si une main restée dans l'ombre, celle de l'écrivain, appuyait sur un bouton de télécommande). La bande son est constituée par la narration de personnages qui se racontent d'une voix égale, monocorde, obéissant à une logique interne au monde représenté, un monde où les individus sont lobotomisés dans une société dominée par l'omniprésence de l'univers médiatique et la prédominance de la logique de consommation. Le profil des personnages mis en scène est volontairement flou et interchangeable, stylisé à l'extrême : cerné par des caractéristiques génériques (homme/femme), un prénom, un âge (ils sont pour la plupart jeunes, entre vingt et trente ans), une profession, et une identité réduite à leur appartenance à un signe zodiacal qui au lieu de les identifier avec plus de précisions ne fait quaccroître leur anonymat. Plus que des personnages à la psychologie fouillée, il serait plus exact de parler à propos des créatures d'Aldo Nove de types rendus aisément reconnaissables à leur registre de langue très bas et à un horizon culturel pauvre. Le personnage aldonovesque est dans la plupart des cas jeune, stupide et vulgaire, doté d'une culture forgée à l'aune des pires émissions télévisées et des spots publicitaires, dépourvu d'idéal et de rêve, incapable d'exister et d'agir autrement que par l'assouvissement de ses instincts primitifs et un comportement irrationnel de consommateur. On le reconnaît à la stylisation de son langage fragmenté en phrases élémentaires et agrammaticales, porteuses d'une vision de la vie et d'une psychologie atrophiées, une langue que Giuseppe Antonelli a identifiée dans un premier temps comme "ipermedia ${ }^{3}$, avant de montrer combien

3. "Un italiano sostanzialmente substandard, popolare e alienato, infarcito di stereotipi e di ossessive ripetizioni" (Antonelli, 2002, p. 40). Voir également Pomilio (1999, p. 675) : "il parlante medio noviano si esprime in 
Nove avait travaillé l'effet caricatural de ses personnages en leur mettant en bouche une "lingua da semialfabeti».

Aldo Nove agite les fils de ses marionnettes humaines nourries aux promotions de l'Esselunga du coin et bercées par des émissions télévisées constituant leur unique horizon culturel dans deux directions satiriques qui se mêlent souvent : s'attachant à une critique impitoyable de la mainmise de la télévision sur l'individu, il condamne en même temps les comportements induits par une société de consommation bêtifiante.

À ces premières observations s'ajoute un constat : Nove, qui a fait ses débuts sous l'étiquette désormais bien connue des cannibali avec son premier récit publié dans l'anthologie Gioventù cannibale (Brolli, 1996), manie la satire avec une palette de tonalités allant de la saynète grotesque aux récits les plus corrosifs. Ainsi, dans Moltissima acqua e un po di sangue, un père de famille des plus honnêtes, désespéré par le cas de son fils possédé par le démon de la télévision, décide dans un premier temps de recourir aux services d'un sorcier pour le libérer. Après d'infructueuses mais surtout très coûteuses manœuvres de désenvoûtement, le père de famille comprend la vénalité du sorcier d'opérette et décide cette fois, par souci d'économie, de se débrouiller par ses propres moyens : après avoir fait ingurgiter de force au jeune homme possédé des litres d'eau salée, il le jette par la fenêtre et lui fait exécuter un vol de plusieurs étages. Si la scène grotesque de cet exorcisme fatto in casa, à mi-chemin entre le splatter et une lointaine commedia all'italiana est l'une des moins sordides du recueil, elle permet toutefois de repérer une stratégie d'écriture reposant sur le contraste, ici entre l'ingénuité d'un personnage fermement convaincu d'agir en toute honnêteté et la gravité du geste qu'il accomplit.

\section{I personaggi di Nove: non sono dei finti cattivi!}

C'est ce contraste que Nove décline et accentue dans ses autres récits au point d'en faire des récits programmés pour être dérangeants car la typologie des personnages mis en scène est le fruit d'un savant cocktail entre naïveté et cruauté comportementale, entre candeur et sordide. Lauteur en avait déjà donné une preuve dans la nouvelle Il mondo dell'amore publiée dans l'anthologie des Cannibali avec deux personnages - qui anticipent le type du personnage woobindien - dotés d'une cruauté sans borne doublée

un italiano televisivo ridotto a un grado prossimo all'inespressività e all'afasia, che lo incolla all'assoluto presente dei suoi bisogni consumistici sottoproletari." 
d'une inconscience puérile au point d'exercer cette violence sur leur propre personne. Voici en substance la teneur du récit : après avoir acheté dans un supermarché de Varese une cassette pornographique dans laquelle est montrée une intervention chirurgicale pour devenir transsexuels, Michele et Sergio, galvanisés par l'alcool et tout excités par la scène qu'ils regardent, par un effet d'imitation des plus infantiles, décident de pratiquer la même opération sur eux-mêmes à l'aide d'un couteau de cuisine. La scène de la castration s'attarde sur leur agonie dans une mare de sang avec Michele, incrédule devant la mort qui se profile avec certitude, et qui tente puérilement de l'éloigner par une comparaison hypothétique typique du monde des contes, "era come se morivo", et est tout surpris de mourir pour de vrai sans renoncer, avant d'expirer pour de bon, à emporter avec lui le souvenir du jeu de toujours, le début de l'émission Ok il prezzo è giusto présenté par Iva Zanicchi. Voici comment il raconte sa dernière heure :

$F u$, quello, l'ultimo sessantanove della mia vita. Il primo da donna. E l'unico da moribondo. Nella mia testa c'era una vera confusione. Sentivo un ronzio, ossessivo, che diventava una specie di musica perfetta. Sentivo come delle risate lontane. Come degli echi indescrivibili. Come se attorno a me c'era molta gente. Come quando Iva Zanicchi entra e c’è quella specie di festa, prima della pubblicità. (Nove, I996, p. 62.)

On demeure pantois devant la violence à l'état brut offerte en pâture dans le récit, même si certains détails relèvent d'un hyper réalisme conduisant à faire basculer le texte dans une forme de violence maniériste qui en limite justement l'impact émotionnel car l'excès de la représentation verse dans la caricature. Ce qui frappe davantage est le fait que Nove puise sa force expressive précisément dans le contraste entre l'analphabétisme de ses personnages et leur excessive compétence dans les marques de produits de consommation et d'émissions télévisées. Le résultat de cette alchimie narrative est terrifiant : la société décrite par Nove est dominée par une humanité grotesque et féroce où les sentiments amoureux sont phagocytés par une érotomanie maladive, les rapports familiaux ont éclaté sous l'impact de la culture audiovisuelle, la vie sociale entière est régie par un rapport de dépendance totale aux objets de consommation, la perspective même du rêve individuel est niée par une subordination totale aux modèles institués par l'univers médiatique. Si l'on peut lire certains récits de Woobinda comme des versions dégradées et caricaturales du rêve romantique ("A letto con Magalli»), de l'idéal de liberté («Woobinda»), ou encore du symbole générationnel («Neocibalgina»), le domaine dans lequel la plume de Nove devient la plus acérée concerne l'impact du monde audiovisuel sur l'individu. En effet, la télévision (et celle qui est visée est plus particulièrement celle de l'ère Berlusconi à en croire le récit "Woobinda») 
façonne les modes de pensée, oriente les désirs, et peut conduire jusqu'au crime. Le récit d'ouverture du recueil - Il Bagnoschiuma - est là pour nous le rappeler. Vidal est cette marque de produit pour le bain, qui était diffusée à la télévision dans les années 1980, dont le flacon rappelle métonymiquement l'essence même du pin sylvestre qui lui donne son parfum. La publicité qui représentait un cheval blanc galopant en liberté sur une plage au son d'une musique inspirée de Laila d'Eric Clapton renvoyait à un style hédoniste et blues. Le personnage de Nove, endoctriné par cette publicité pour Vidal, voyant que ses parents utilisent une autre marque par souci d'épargne, entre dans un déni de la réalité qui le conduit au violent parricide. Le récit fait à la première personne permet une approche intradiégétique du crime et conduit le personnage à se justifier et à légitimer son acte après l'avoir commis en se faisant fort d'une logique de consommation toute personnelle :

Ho ammazzato i miei genitori perché usavano un bagnoschiuma assurdo, Pure e vegetal. Mia madre diceva che quel bagnoschiuma idrata la pelle ma io

Uso Vidal e voglio che in casa tutti usino Vidal.

Perché ricordo che fin da piccolo la pubblicità del bagnoschiuma Vidal mi piaceva molto. Stavo a letto e guardavo correre quel cavallo.

Quel cavallo era la libertà, volevo che tutti fossero liberi.

Volevo che tutti comprassero il Vidal. (Nove, I996, p. II.)

Le récit d'ouverture constitue une véritable clef de lecture du recueil Woobinda: il pose les jalons d'un glissement sémantique qui est à la base d'une logique, celle d'une stratégie communicative spécifiquement publicitaire que dénonce Nove tout en la métabolisant dans son écriture, comme a pu le souligner avec brio Rita Gagliano :

Non il prodotto dozzinale costituito dal Pure e Vegetal bensì il bagnoschiuma Vidal si carica di significazioni che vanno al di là del suo uso effettivo e immediato attraverso un dispositivo metonimico adottato dalla stessa pubblicità nostalgicamente evocata dal protagonista. Il marchio, arricchito di un significato ideale e astratto imposto dalla sua contiguità con quel cavallo bianco viene a sostituire il termine generico che di conseguenza perde il suo statuto di segno. [...] il marchio di fabbrica, innalzato a ideale assoluto, impone il suo potere egemonico sugli oggetti e sui corpi. La merce e il crimine sono legati da una falsa logica associativa nella quale il marchio Vidal non rappresenta ma si identifica senza mediazione metaforica con un ideale di libertà di acquisto, la cui ostruzione non può che essere ovviata dalla violenta e fredda rimozione dei suoi due ostacoli. I genitori stessi sono vittime di quello svuotamento semantico del termine generico del bene commerciale che li riduce a meri oggetti, non piu relazionabili al protagonista proprio a livello di scelta di consumo. Cade ogni legame affettivo o familiare, come base fondante dell'io, sostituito dal feticismo merceologico. (Gagliano, 200I, p. 29I.) 
Le texte s'inscrit dans un processus de consommation qui est non seulement la cause formelle du crime mais aussi le principe même d'organisation chronologique interne : au-delà des perceptions temporelles du personnage (littéralement condensées autour des actes de consommation qui scandent ses journées apparaissant comme un flux ininterrompu de consommation), Aldo Nove n'hésite pas à s'approprier des codes linguistiques de marketing ${ }^{4}$ et à les ingérer textuellement.

Cependant, au-delà de ces analyses, il convient de se recentrer sur les conséquences du processus de réification des personnages woobindiens : l'une d'elles est la perception altérée de la réalité. En effet, à partir de son engouement pour la marque Vidal, le protagoniste se recrée un réel virtuel dans lequel il se réfugie, symbolisé par des objets et des marques («mangiavo in camera mia, patatine e tegolini del Mulino"), et qui finit par se substituer au réel de son existence avant de devenir la justification d'un double meurtre.

Ce renversement du système de valeurs explique pourquoi les personnages de Nove sont d'une violence inouïe, précisément parce que leur substrat culturel - qui est le socle de leur logique et préside à leurs agissements - nous est familier, ce qui a fait dire sur un ton provocateur à Tommaso Labranca :

Aldo Nove è il principe dei realisti [...] è un realista perché il suo delitto nasce da una situazione verificata nella realtà, conseguente all'acquisto di un detersivo offerto a prezzo inferiore. Violenza non come patologia aberrante, ma come ultimo anello di una catena di violenze acutamente concorrenziali che definiscono la libera economia di mercato. [...] Nella sua narrazione ci sono personaggi monodimensionali e normodotati, assolutamente dimenticabili, che ai critici cialtroni possono sembrare mostri aberranti e invece sono i nostri normali vicini di casa nel condominio della realtà. (Labranca, 1998, p. I4I.)

Nove n'agite pas devant nos yeux ces finti cattivi qui furent tant fustigés par Filippo La Porta ${ }^{5}$, censés divertir les lecteurs par une imagination débordante, à coup de litres de sang ou d'autres humeurs versées, et de mutilations en tout genre. Les personnages de Nove ne font pas rire, ils dérangent. Ils participent d'un paysage humain où certes, les actions

4. On pensera en particulier au cas des Weasel words (Gagliano, 200I).

5. Pour Filippo La Porta (Non c’è problema, 1997) les auteurs de Pulp italiens ne sont pas assez audacieux dans leur assimilation du trash. Ils se réfugieraient dans un style horrifique maniériste à défaut de trouver un langage adéquat. L'idée trouve son prolongement dans le concept de "travestimento all'italiana» qui guide l'approche critique de F. La Porta. Dans l'un de ses précédents ouvrages, La nuova narrativa italiana. Travestimenti e stili di fine secolo (1995), il avait déjà souligné le manque d'imagination des auteurs pulp. À son tour, Gianni Canova ("Il fantasma dell'Horror", Tirature 'g8), revient avec insistance sur l'incapacité à raconter l'horreur en insistant sur la propension italienne à transformer "la paura in spauracchio comico e a sghignazzare felici». 
se traduisent par des actes violents marquant une indéniable si ce n'est directe filiation au Pulp ${ }^{6}$, mais où les motivations qui les guident prennent racine précisément dans un terreau bien concret et identifiable. Or c'est précisément sur cette voie que progresse la narration de Nove dans Woobinda au point de renverser les données du réel, de les subvertir de façon inéluctable au point que l'univers télévisuel finit par se substituer au réel et à être plus vrai et plus crédible que le réel.

Le récit intitulé «Vermicino» est particulièrement éloquent à ce propos. Il s'agit d'un fait divers horrible ayant réellement défrayé la chronique italienne en I98I : cette année-là, Alfredo Rampi, un jeune garçon âgé de six ans, tombait au fond d'un puits artésien plongeant à 30 mètres dans les entrailles de la terre à Vermicino. Cet événement eut une importance médiatique particulière : il s'agit du premier cas de fait divers, longuement retransmis à la télévision, à avoir tenu en haleine des millions de personnes devant leur écran pour suivre les opérations de sauvetage qui durèrent trois jours.

Aldo Nove revisite cet événement dramatique en montrant la monstruosité induite par un pouvoir cathodique ravageur : pour avoir eu l'opportunité de regarder l'agonie en direct, son personnage a le sentiment d'avoir vraiment connu une expérience inoubliable que seule la télévision a pu lui permettre de vivre en raison de sa capacité à transformer l'événement dramatique individuel en expérience collective. Dans cette perspective, la participation émotive au drame de l'enfant devient pour le téléspectateur une façon de s'inscrire dans l'Histoire italienne, d'en faire partie intégrante car cette fois, la télévision aimante son attention en lui faisant vivre par procuration l'indicible, ce qui constitue à la fois le plus grand mystère et la plus grande inquiétude de l'existence, à savoir la Mort :

$\grave{E}$ una cosa importante. É forse la cosa più importante, avere qualcosa da ricordare come Vermicino.

Un fatto che ti è accaduto, e che se vai a cercare lo ritrovi intatto, messo a posto via dentro te stesso. Cosi se cerchi di ricordare ti fermi, c’è qualcosa di solido, che rimane. Da raccontare ai tuoi nipoti. La storia.

Questo Vermicino, io lo ricordo.

Perché forse è stato il momento più bello della mia vita, te lo racconto cosi come è successo, con la luce spenta tutti alzati assieme a guardarlo. C'era silenzio. Era notte. Diventava sempre più notte a guardare Vermicino alla tele. Eravamo milioni di persone e lui, giu, li da solo.

6. Le film culte faisant office de référence est Pulp Fiction de Quentin Tarentino, États-Unis, I994. 
Cercava di non morire, con un microfono lo diceva a tutti $i$ telespettatori, che non voleva morire Alfredino Rampi. E noi, li, come dei tifosi della vita, ad aspettare che si vedesse che lo salvavano da quel buco.

Mia madre diceva fate silenzio, fate silenzio tutti si sente che dice qualcosa al microfono, lo intervistavano in silenzio su com'era morire li, senza che nessuno ti può vedere, tutti ad ascoltare però. Se piangeva se gridava. (Nove, 1996, p. 27.)

On ne manquera pas de noter une frustration infantile du personnage qui entend l'agonie plus qu'il ne la voit - et soulignons au passage que le recueil entier de Woobinda privilégie les perceptions auditives. Or, l'image concrète, physique, de l'enfant agonisant est figée dans l'esprit du personnage dans une photographie dont le sujet a des contours flous, atemporels alors qu'un autre type de perception se fait jour en lui progressivement, entièrement focalisée sur le rythme des publicités télévisées qui constituent sa seule référence dans le champ de l'expérience, en somme l'unique vraie scansion temporelle :

Mi ricordo l'espressione di Alfredino, sui giornali sempre quella, che chiudeva un pò gli occhi per il sole, con una canottiera a righe. Prima che cadesse nel fosso della televisione. Allora quando viveva cosi, un bambino normale, anche molto carino era senza la diretta notturna.

Penso che se Alfredino moriva ora aveva la pubblicità come problema, più per $i$ telespettatori che per lui direttamente, impegnato a sopravvivere un attimino in piu. Avrebbero cercato un momento neutro per mandare la pubblicità dei croccanti per il cane, come quando nelle partite la palla esce dal campo, un giocatore va a ricuperarla, e fanno lo spot di una cosa.

Ma moriva sempre allo stesso modo, non c'era pausa, quel bambino moriva tutta la notte. (Nove, I996, p. 28.)

La monstruosité est évidente : seule l'absence de pauses publicitaires ou tout du moins leur renvoi à un moment ultérieur permet au personnage de prendre conscience de la réalité concrète de la mort. À cette constatation s'ajoute une réflexion portant sur la place de la victime dans la représentation mentale de l'événement par le personnage : le Sujet n'est plus, il est réduit à n'être qu'un élément vidé de son humanité, un signifiant quelconque dans une chaîne communicative, un instrument servant de faire-valoir pour accéder à la notoriété.

Per farti intervistare dovevi essere suo parente, o una maestra che lo aveva avuto. Due parole al telegiornale e via, tornavi nessuno.

Alcuni provano a scendere nel buco. [...] Mi sembra che c'era anche il Presidente della Repubblica, che allora forse era Pertini, e stava intorno al fosso come il sindaco di Vermicino.

Per stare vicino al fosso, dovevi essere importante, gli altri guardavano alla tele, come alla Scala, se non sei qualcuno vai su nel loggione. (Nove, 1996, p. 28.) 
Dans l'optique de ce monologue conduit avec le langage typique d'un esprit gangrené par les expressions courantes du monde télévisuel (on pensera en particulier à cette expression que Nove met souvent en bouche à ses personnages, "un attimino", qui a remplacé dans le langage courant "un poco» ${ }^{7}$ ), il existe une vie avant il fosso et une après, sachant que la réitération du terme fosso indique qu'il devient le catalyseur d'une fascination sans bornes pour l'objet télévision qui a le pouvoir de rendre visible l'existence de l'individu, et donc de le faire exister tout court. Regarder le réel par le prisme de la télévision revient à transformer la mort en spectacle réel, à la crédibiliser ${ }^{8}$, à faire entrer ses victimes au panthéon des célébrités, à propulser les assistants impuissants de la scène au rang des personnalités (d'ailleurs le personnage ne se souvient plus très bien du nom du Président de l'époque, de même que le maire n'est pas indiqué comme une personne ayant une identité définie, seule compte leur proximité au fosso). Parallèlement, le reportage télévisé dans sa matérialité se substitue dans la conscience du personnage à l'événement réel précisément parce qu’il le relaie en direct sans discontinuer, abolissant de ce fait toute barrière temporelle entre le fait et sa perception :

Vermicino era un programma davvero spontaneo. Non come certi programmi di adesso, ad esempio Perdonami di Mengacci, dove è già successo che hanno ammazzato veramente, ma non era come a Vermicino. (Nove, 1996, p. 29.)

Un degré de plus dans la confusion entre la réalité et sa représentation est franchi avec le récit «La strage di via Palestro ${ }^{9}$ » où les personnages, qui ont été des témoins oculaires de l'attentat, ont besoin de le voir retransmis à la télévision pour y croire. Écoutons plutôt :

Quando sono andato alla strage di via Palestro con la mia ragazza, Capricorno, era vestita in modo da puttana.

7. Filippo La Porta analyse la portée sociologique et symbolique de l'expression "un attimino" faisant remarquer : "La parola "attimino" si è proditoriamente sostituita nel linguaggio corrente a "un poco", "un po". Una categoria temporale (e in una sua forma diminutiva, vezzeggiativa, che può ricordare lo spagnolo un momentito) ha scacciato una categoria spaziale. Dunque non si dirà più "un po' meno aggressivo", ma "un attimino meno aggressivo", variante impazzita: "un attimissimo" sentito da Lucia Annunziata nel corso della trasmissione Prima serata. " (La Porta, I997, p. 49.)

8. Dans le même ordre d'idées, ne trouve-t-on pas dans nos boîtes à lettres des prospectus vantant les vertus de tel ou tel autre objet avec la mention "vu à la télé» pour asseoir la crédibilité de la publicité et faire franchir le pas au consommateur?

9. "Alle 23.I4 del 27 luglio I993, in via Palestro a Milano, una Fiat Punto saltò in aria davanti al Padiglione d'arte contemporanea. Un attentato di stampo mafioso in cui morirono Moussafir Driss, marocchino, che dormiva su una panchina, tre vigili del fuoco - Stefano Picerno, Carlo La Catena e Sergio Pasotto - e il vigile urbano Alessandro Ferrari, intervenuti perché da quell'auto usciva fumo. Dodici i feriti, sventrato il Padiglione d'arte contemporanea." 
Ciò poteva sembrare irrispettoso, specialmente perché aveva i pantaloni aderenti, neri, ma nessuno sembrava farci caso perché davanti a una strage non si fa caso nemmeno alla figa.

Prima la sera uno è normale, magari è tuo marito, o tua moglie, e poi va in via Palestro e finisce a tocchetti sugli alberi e per terra e sui cofani delle auto parcheggiate duecento metri più in là, e non si trova ad esempio un pezzo di schiena, e quello era tuo marito dentro i sacchetti per $i$ morti.

Tutti pensano ai morti. Anch'io. Ho vent'anni. Passando tra la gente, mi vedevo le macerie ed ero triste, ma meno che guardando la televisione, perché alla televisione tutto sembra più vero, e i collegamenti sono immediati, la strage ti entra in casa all'improvviso, non c’è calcolo, nessuno dice "andiamo alla strage», succede. (Nove, 1996, p. 33.)

La télévision n'est plus un filtre permettant d'accéder au réel à travers une représentation, elle est ce réel. Dans ce même esprit, quand Nove ne montre pas les crises absurdes de boulimie consommatrice déclenchées par la nouvelle du déclenchement de la guerre en Irak ("Baghdad»), il érige la télévision au rang de deus ex machina de la modernité («Protagonisti»). Le principe de cette émission repose sur une inversion totale des rôles des spectateurs et des acteurs : pendant le show télévisé, le spectateur devient précisément, le protagoniste. En voici un extrait :

Mi chiamo Matteo Pirovano e ho ventidue anni. Appartengo al segno dell'Aquario. Pur avendo elaborato interessanti dottrine cosmologiche, fino a poche settimane fa la mia vita scorreva come qualcosa di estraneo, era un mistero di cui non riuscivo a trovare la soluzione. Per questo andavo male all'università. Per questo non riuscivo a trovare una ragazza. Ora le cose cambiano a una velocità di cui io stesso mi stupisco. Ora guardo sempre Protagonisti. Va in onda ogni giorno alle diciannove. Pubblico che ti guarda, nient'altro. Pubblico competente, bella gente: Protagonisti è il programma che ti mette al centro della scena. Protagonisti è il vettore attraverso il quale ogni giorno il mio successo sincunea nel cuore della gente. Quando termina la sigla iniziale e le oltre trecento facce di esperti e di belle ragazze mi guardano dallo schermo inizio a parlare. Quelli mi seguono interessati e snocciolo le mie teorie con la consapevolezza di quanto io stesso valga. (Nove, 1996, p. 136.)

La télévision, en allumant les projecteurs sur les individus lambdas, sur leurs misères quotidiennes et leurs peines existentielles, panse leurs plaies, guérit leurs complexes, atténue leur mal de vivre, leur permet d'affirmer leur vraie personnalité : elle devient le moyen d'échapper à la grisaille de l'anonymat, à la tristesse de l'ordinaire pour proposer comme alternative l'illusion d'une vie correspondant aux vœux les plus secrets, une image virtuelle de soi, qui gomme la médiocrité, elle, bien concrète, des individus.

On voit bien sur quel terrain Nove choisit d'évoluer afin de s'attaquer aux multiples formes de l'aliénation télévisée qui caractérisent la société de masse et de consommation : il procède à une critique de l'intérieur de 
cette même société, après en avoir étudié, ingurgité et adopté les formes langagières, les registres de langue, les stratégies communicatives.

On pourrait voir dans cette forme de cannibalisme un mimétisme langagier et comportemental fonctionnant comme un miroir de la société, renvoyant à ses excès, à ses turpitudes, à ses malaises si ce n'est que les palettes du grotesque et de la caricature, maniées avec dextérité par Nove, brouillent cette piste interprétative. En effet, s'il est vrai que le procédé de base de la satire est la dégradation - et cela est vrai dans le cas de Woobinda tant au niveau du contenu que du style et de la langue -, dès lors que l'auteur laisse planer le doute sur la nature de ses intentions et de son ironie militante, on est en droit de percevoir des limites aux formes d'expression qu'il a choisies ${ }^{\text {IO }}$.

\section{Les limites : Superwoobinda et Puerto Plata Market}

Les premiers éléments troublants sont repérables dans Superwoobinda, c'est-à-dire la réédition en 1998 de la totalité des textes contenus dans Woobinda avec l'ajout de douze micro-récits. Si l'on y retrouve la composante corrosive et polémique qui avait fait le succès des récits woobindiens, on s'aperçoit cependant que le côté pulp de l'écriture y est plus chargé, la vulgarité plus accentuée, l'imagination plus débridée au point que certains récits, comme il Sol dell'avvenir sont composés comme un délire mêlant violence, pornographie, critique politique et misère humaine. Ce qui surprend le plus à deux ans de distance de Woobinda, c'est la façon dont Aldo Nove qui, jusqu'alors avait été une présence auctoriale effacée se contentant de zapper sur les tranches de vie de ses personnages, se met à présent en scène.

Dans «Tre racconti sulla televisione», Aldo Nove apparaît aux côtés de ses créatures ${ }^{\text {II }}$ en pleine régression langagière pour se mettre au niveau de ses personnages et raconte sur un ton volontairement benêt et goguenard comment il a été tiré d'un onanisme reconstituant par un coup de fil l'invitant à participer à une émission télévisée. C'est l'occasion d'une autodérision qui repose sur l'intertextualité (Nove se sert du succès de Woobinda

Io. On se reportera à l'opinion de Northrop Frye, Anatomie de la critique, [Princeton University Press, I957], Paris, Gallimard, I969 : «La satire est une ironie militante, ses normes sociales sont relativement claires, elles lui permettent de prendre la mesure du grotesque et de l'absurde. Quand le lecteur par contre n'est pas très sûr des intentions de l'Auteur, l'ironie ne comporte guère d'élément satirique.» (p. 272)

II. Il se présente ainsi en ouverture du récit : "Mi chiamo Aldo Nove, ho ventinove anni e sono uno scrittore con il quale le ragazze che vanno più d'accordo sono quelle dei segni del I) Toro;2) Vergine; 3) Cancro." 
qui devient à son tour une matière première de recyclage pour l'écriture), comme par exemple dans cet extrait qui est un clin d'œil explicite au récit «Protagonisti» évoqué précédemment :

Quando le telecamere cominciano ad inquadrarti allora sei uno scrittore. Uno scrittore senza televisione fa sghignazzare da mattina a sera, per dirla tutta lo scopo degli intellettuali che sono vincenti è andare sempre all'Altra edicola, una simpatica trasmissione di cultura che fanno vedere il giovedi sera su quel canale che è Raidue. (Nove, 1998, p. 136.)

L'autodérision se transforme très vite en autodégradation : de clins $\mathrm{d}^{\prime} \mathrm{Ceil}^{\mathrm{12}}$ en allusions, il joue à se glisser dans la peau d'un inferior auctor ${ }^{13}$ à l'humeur satyriasique, adoptant le langage et l'horizon d'attente de ses propres personnages :

Dopo che ho scritto Woobinda, mi hanno invitato a una trasmissione di cultura dove c'era Bevilacqua, Cortocircuito. Io ero emozionato perché voglio diventare il Bevilacqua del Duemila. Bevilacqua, quando parla è pensieroso. Sempre inizia a gridare contro qualcuno, perché è focoso. Anchio voglio così, ma per adesso non ce la faccio siccome che ho questo problema della timidezza. Quella volta, c'era anche Selen, la pornostar che a me piace ma non ha fatto neanche un pompino. (Nove, 1998, p. 140.)

À ce point de notre analyse, il convient de s'interroger sur les conséquences narratives de cette tendance de l'auteur à créer et à entretenir la confusion avec le sujet de sa représentation. Nove semble ne pas vouloir exercer cette distance critique par rapport à son sujet ${ }^{\mathrm{I} 4}$, garante du bon fonctionnement de la satire, comme s'il était soudain happé par les mécanismes du monstre qu'il avait engendré. Dans Woobinda, la main de l'auteur, restée dans l'ombre, zappait sur une humanité dégradée mais dont la dégradation, précisément parce qu'elle était montrée dans toute sa cruelle nudité, constituait en soi une dénonciation implicite, un outil critique tout aussi implacable qu'efficace. Le montage narratif et textuel, sous forme de pot-pourri télévisuel symbole d'une génération bien identifiable, pouvait permettre une lecture éthique de l'œuvre, dans le sens que lui attribue Alberto Casadei ${ }^{15}$, c'est-à-dire une éthique repérable dans la

I2. «Ciao, sono Aldo Nove, lo scrittore che piace» (Nove, 1998, p. I53) ou encore "Pulp? ancora!? Basta!» (Nove, I998, p. I8I).

13. Pour le critique Massimo Arcangeli, il s'agit d'une forme de "narcissismo infantile» (Arcangeli, 2007).

14. Pour T. Pomilio (1999, p. 678) : "L'etica transcendentalmente popolare di Nove è quella invece, di prodursi in quanto autore inferiore, proprio contro ogni riscatto stilistico, e prodursi, poi, in favore di quella confusione di autore e personaggio. Perché per Nove, la sola etica veramente possibile in letteratura è quella di non produrre stile in quanto marca autoriale di separazione, quella separatezza dell'artista e autore, che fu l'ultima farragine e riscatto di Tondelli."

15. Pour A. Casadei (2007, p. II9) : "L'etica narrativa deve essere esaminata in primo luogo sulla base dell'intreccio, ossia delle implicazioni che la trama riesce a proporre in quanto organizzazione testuale di comportamenti 
construction même de l'œuvre et non pas estimée à l'aune de comportements interprétables selon les lois morales ou juridiques en vigueur. Nove frappait du poing par une langue appauvrie, des personnages abjects, il nous choquait, nous bousculait par un malaise interrogateur, il réveillait la conscience de ses lecteurs.

Mais dès lors que l'Auteur se propulse au rang de la matière dégradée qu'il observe, la force expressive de la satire perd en force et originalité, et l'écriture verse dans la farce grand-guignolesque rendue au moyen de la dilatation de procédés narratifs et stylistiques qui avaient été les attributs de son style novateur au temps de Woobinda. Pour en juger, il suffit de jeter un regard furtif au premier roman de l'auteur paru précisément entre Woobinda et Superwoobinda et qui amorce déjà le procédé de dilatation : dans Puerto Plata Market, sorte de roman de formation sentimentale, le personnage de Michele, après une déception sentimentale, entreprend un voyage à Saint Domingue, destination très prisée du tourisme sexuel pour tenter d'y trouver l'âme sœur. Les scènes du séjour en République dominicaine sont entrecoupées de souvenirs d'enfance car le protagoniste, énième alter ego des consommateurs woobindiens, est demeuré prisonnier d'une enfance dorée, bercée par les dessins animés et les émissions de variété, une enfance mythifiée, associée invariablement aux objets de consommation variés qu'il aimait se procurer dès son plus jeune âge. Pour écrire son roman, Nove a repris des éléments de ses courts récits (humour vulgaire à double sens érotique, candeur excessive du personnage, habitudes consommatrices forcenées) qu'il a amplifiés au point que l'on trouve des pages entières de listes de produits énumérés (le plus long et le plus irritant va de la page 59 à la page 63$)^{16}$.

Comment interpréter cette auto-dégradation volontaire, cette descente aux enfers narrative qui ne rachète rien ni personne? Le mimétisme de la figure auctoriale avec son Sujet est-il le signe d'une aporie stylistique indiquant que l'auteur a été gangrené par cette même société qu'il avait regardée et zappée dans Woobinda? Nous aimerions pencher pour l'hy-

umani messi in scena nella sfera del possibile e non dell'accaduto, e quindi non immediatamente riducibili al loro puro contenuto esplicito e informativo. In altre parole mentre risulta forzata ogni interpretazione etica di un romanzo che si fondi sulla mera fabula e che conduca a una sorta di precettistica, una considerazione attenta della costruzione testuale può proporre domande non scontate sull'etica possibile manifestata dalla trama."

I6. Un autre exemple de reprise amplifiée et de déjà-vu, lorsqu’il reprend une idée exprimée par l'ensemble du recueil Woobinda, celle consistant à préférer les "fighe dei giornali a quelle vere» : «il funzionamento delle cose che sono immediatamente vita pensandoci bene mi sembra molto volgare, e allora a me vanno senz'altro bene tele e finzione assoluta." (Nove, I997, p. I00.) 
pothèse d'une instrumentalisation pleinement consciente de la matière par Nove, mais ce dernier persiste à vouloir faire rire par la médiocrité et l'avilissement avec des personnages qui tiennent à la fois de Forrest Gump ${ }^{17}$ et Charles Bukowski. Le problème est que les clins d'œil adressés aux lecteurs et les multiples tentatives de le divertir par la mascarade opèrent un déplacement de l'acte de réception : à patauger dans une dégradation dans laquelle il se complait, Nove non seulement anesthésie l'esprit critique mais devient complice de la réification et de l'aliénation qu’il dénonçait.

\section{Bibliographie}

Antonelli Giuseppe, Lingua ipermedia. La parola di scrittore oggi in Italia, Lecce, Manni, 2006.

—, "La lingua della narrativa italiana degli anni Novanta», Sguardo sulla lingua e la letteratura italiana all'inizio del terzo millennio, Bruxelles, mars 2002, Atti del convegno a cura di Sabina Gola e Michel Bastiaensen, Firenze, Cesati, p. 37-6I.

Arcangeli Massimo, Giovani scrittori, scritture giovani, Rome, Carocci, 2007.

Barenghi Mario, Oltre il Novecento, Milan, Marcos Y Marcos, 1999.

Bazzocchi Marco Antonio, Corpi che parlano, Milan, Bruno Mondadori, 2005.

Brolli Daniele, Gioventù cannibale, Turin, Einaudi, coll. "Stile libero», I996.

Canova Gianni, "Il fantasma dell'horror», Tirature '98, a cura di Vittorio Spinazzola, Milan, Mondadori, 1997, p. 86-89.

Carnero Roberto et Ladolfi Giuliano, Sentieri narrativi del novecento, Novara, Interlinea, $200 I$.

CASAdei Alberto, Stile e tradizione del romanzo italiano contemporaneo, Bologne, Il Mulino, 2007.

Cerasi Enrico, "Sublime di massa no grazie», L'Indice dei libri del mese, $\mathrm{n}^{\circ} 3, \mathrm{I} 997$.

17. Il s'agit du film de Robert Zemeckis, d'après le roman Forrest Gump. Selon Mario Barenghi, «Woobinda di Aldo Nove con Tarentino, non ha molto a che spartire. [...] In Italia, una Pulp Fiction non esiste. Non esiste una letteratura (letteratura non cinema o fumetto o altro) popolare, sensazionalistica e di successo, suscettibile di rielaborazione colta. Non esiste un equivalente dei film di terzo o quarto ordine che Tarentino usa parodiare. Dunque l'oggetto di questa parodia non può che essere extraletterario, ovvero non italiano" (Barenghi, I999, p. 279). 
De Federicis Lidia, «Poetiche e palestre», L'Indice dei libri del mese, $\mathrm{n}^{\circ} 6$, I998.

FrYe Northrop, Anatomie de la critique, [Princeton University Press, 1957], Paris, Gallimard, 1969.

GaGliano Rita, "Il protagonista-consumatore e la merce-segno: il percorso iper-consumistico di Aldo Nove nel Bagnoschiuma", Narrativa, $\mathrm{n}^{\circ}$ 2O-2I, juin 200I, p. 285-295.

Giacovelli Enrico, La commedia all'italiana. La storia, i luoghi, gli autori, gli attori, i film, Rome, Gremese editore, [1990], 1995.

Hodgart Matthew, La satire, Paris, Hachette, 1969.

Hofmeister Alexandra, "Dentro la televisione ci siamo noi», Horizonte, $\mathrm{n}^{\circ}$ 6, 200I, p. 63-89.

Labranca Tommaso, Chaltron Hescon, Turin, Einaudi, coll. «Stile libero», I998.

La Porta Filippo, La nuova narrativa italiana. Travestimenti e stili di fine secolo, Turin, Bollati Boringhieri, 1995 .

—, Non c’e problema, divagazioni morali su modi di dire e frasi fatte, Milan, 1997.

Lucamante Stefania, Italian Pulp Fiction, The New Narrative of the Giovani Cannibali Writers, Londres, Associated UP, $200 \mathrm{I}$.

Nove Aldo, Woobinda e altre storie senza lieto fine, Castelvecchi, [1996], 2005 .

—, "Il mondo dell'amore», Gioventù cannibale, a cura di Daniele Brolli, Turin, Einaudi, coll. «Stile Libero», 1996.

-, Puerto Plata Market, Einaudi, 1997.

-, Superwoobinda, Einaudi, [1998], 2006.

Pasolini Pier Paolo, Saggi sulla politica e sulla società, Milan, Mondadori, coll. «I Meridiani», 1999, 2024 p.

-, Contre la télévision, Besançon, Les Solitaires Intempestifs, 2003.

Pezzarossa Fulvio, C'era una volta il pulp. Corpo e letteratura nella tradizione italiana, Bologne, Clueb, 1999, $234 \mathrm{p}$.

Pischedda Bruno, "Postmoderni di terza generazione», Tirature ' 98 , a cura di Vittorio Spinazzola, Milan, Mondadori, 1997, p. 4I-45.

Pomilio Tommaso, «Le narrative generazionali dagli anni Ottanta agli anni Novanta", Storia generale della letteratura italiana, vol XII, I999.

Sinibaldi Marino, Pulp, La letteratura nell'era della simultaneità, Rome, Donzelli, 1997.

Spinazzola Vittorio, "Gli integrati di Aldo Nove», L'Indice dei libri del mese, $\mathrm{n}^{\circ} \mathrm{I}, \mathrm{I} 999$. 
Turchetta Gianni, «I cannibali non mordono più », Tirature 2005, a cura di Vittorio Spinazzola, p. Io-I7.

Yehoshua Abraham, Comment construire un code moral sur un vieux sac de supermarché. Éthique et littérature, [Tel Aviv, 1999], Paris, Éditions de l'Éclat, 2004.

Intervista a Aldo Nove : <http://www.wuz.it/archivio/cafeletterario.it/ interviste/nove.html>. 
